\title{
Identification and properties of proteases from an Acanthamoeba isolate capable of producing granulomatous encephalitis James Sissons ${ }^{1}$, Selwa Alsam ${ }^{1}$, Graham Goldsworthy ${ }^{1}$, Mary Lightfoot ${ }^{1}$, Edward L Jarroll ${ }^{2}$ and Naveed Ahmed Khan*1
}

\author{
Address: ${ }^{1}$ School of Biological and Chemical Sciences, Birkbeck College, University of London, England, UK and ${ }^{2}$ Department of Biology, \\ Northeastern University, Boston, MA, USA \\ Email: James Sissons - j.sissons@bbk.ac.uk; Selwa Alsam - s.alsam@bbk.ac.uk; Graham Goldsworthy - g.goldsworthy@bbk.ac.uk; \\ Mary Lightfoot - mlightfoot@bbk.ac.uk; Edward L Jarroll - e.jarroll@neu.edu; Naveed Ahmed Khan* - n.khan@sbc.bbk.ac.uk \\ * Corresponding author
}

Published: 03 May 2006

BMC Microbiology2006, 6:42 doi:10.1/86/147|-2/80-6-42

This article is available from: http://www.biomedcentral.com/I47I-2/80/6/42

(c) 2006Sissons et al; licensee BioMed Central Ltd.

This is an Open Access article distributed under the terms of the Creative Commons Attribution License (http://creativecommons.org/licenses/by/2.0), which permits unrestricted use, distribution, and reproduction in any medium, provided the original work is properly cited.
Received: 15 March 2006

Accepted: 03 May 2006

\begin{abstract}
Background: Granulomatous amoebic encephalitis due to Acanthamoeba is often a fatal human disease. However, the pathogenesis and pathophysiology of Acanthamoeba encephalitis remain unclear. In this study, the role of extracellular Acanthamoeba proteases in central nervous system pathogenesis and pathophysiology was examined.
\end{abstract}

Results: Using an encephalitis isolate belonging to TI genotype, we observed two major proteases with approximate molecular weights of I50 KD and I30 KD on SDS-PAGE gels using gelatin as substrate. The I $30 \mathrm{KD}$ protease was inhibited with phenylmethylsulfonyl fluoride (PMSF) suggesting that it is a serine protease, while the $150 \mathrm{KD}$ protease was inhibited with I, I0-phenanthroline suggesting that it is a metalloprotease. Both proteases exhibited maximal activity at neutral $\mathrm{pH}$ and over a range of temperatures, indicating their physiological relevance. These proteases degrade extracellular matrix (ECM), which provide structural and functional support to the brain tissue, as shown by the degradation of collagen I and III (major components of collagenous ECM), elastin (elastic fibrils of ECM), plasminogen (involved in proteolytic degradation of ECM), as well as casein and haemoglobin. The proteases were purified partially using ion-exchange chromatography and their effects were tested in an in vitro model of the blood-brain barrier using human brain microvascular endothelial cells (HBMEC). Neither the serine nor the metalloprotease exhibited HBMEC cytotoxicity. However, the serine protease exhibited HBMEC monolayer disruptions (trypsin-like) suggesting a role in blood-brain barrier perturbations.

Conclusion: Overall, these data suggest that Acanthamoeba proteases digest ECM, which may play crucial role(s) in invasion of the brain tissue by amoebae.

\section{Background}

Acanthamoeba are opportunistic protozoans that are widely distributed in the environment. Given the opportunity and the host immune status, pathogenic Acan- thamoeba can invade the human central nervous system (CNS) and produce fatal granulomatous encephalitis [14]. Acanthamoeba granulomatous encephalitis (AGE) is characterized by headache, fever, behavioural changes, 

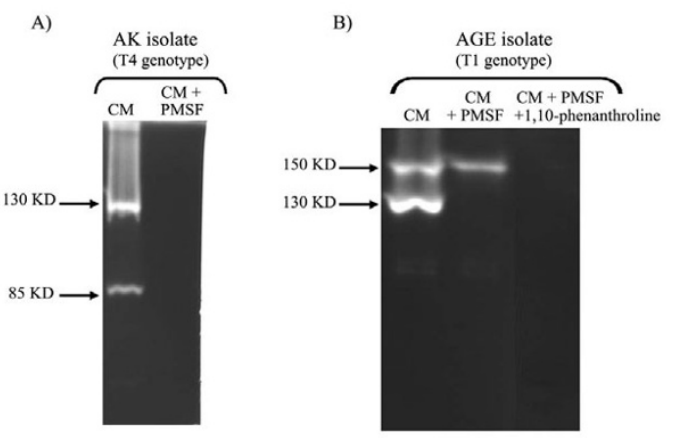

\section{Figure I}

Acanthamoeba extracellular protease profiles of T I (AGE isolate) and T4 (AK isolate). Conditioned medium (CM) from TI and T4 isolates was collected and used for zymography as described in "Materials and Methods". CM + PMSF or CM + I, I0-phenathroline means that the CM was incubated with these protease inhibitors prior to electrophoresis and in the case of $\mathrm{I}, 10$ phenanthroline it was also included in the developing buffer. Note that AK isolate (T4 genotype) exhibited two serine proteases of approx. Mw. of $85 \mathrm{KD}$ and I $30 \mathrm{KD}$ that were inhibited with PMSF, i.e., serine protease inhibitor. In contrast, AGE isolate exhibited one serine protease at approx. Mw. I30 KD and a I50 KD metalloprotease sensitive to I, I0-phenanthroline, i.e., metalloprotease inhibitor. Results are representative of three independent experiments.

hemiparesis, lethargy, stiff neck, aphasia, ataxia, vomiting, nausea, cranial nerve palsies, increased intracranial pressure, seizures and ultimately death. Death is due to haemorrhaging necrotic lesions with severe meningeal irritation and encephalitis. The lesions due to AGE are most numerous in the basal ganglia, midbrain, brainstem, and cerebral hemispheres with characteristic lesions in the CNS parenchyma resulting in chronic granulomatous encephalitis.
The mechanisms associated with the pathogenesis of AGE remain incompletely understood, however the pathophysiological complications involving the CNS most likely include induction of the pro-inflammatory responses, invasion of the blood-brain barrier and the connective tissue and neuronal damage leading to the brain dysfunction [1-4]. The routes of entry include lower respiratory tract leading to amoebae invasion of the intravascular space, followed by the haematogenous spread. Skin lesions may provide direct amoebae entry into the bloodstream, thus bypassing the lower respiratory tract. Amoebae entry into the CNS most likely occurs at the sites of the blood-brain barrier [1,2]. In addition, olfactory neuroepithelium provides another route of entry into the CNS and has been studied in experimental models $[1,2]$. Following CNS invasion, amoebae penetrate the brain tissue to produce disease. Previous studies have shown that proteases play important roles in microbial pathogenesis including host cell and tissue invasion, migration, catabolism of host proteins, cytoadherence, and both stimulation and evasion of host responses [reviewed in [7]]. The present study examines Acanthamoeba proteases released extracellularly by an AGE isolate to begin to ask if these proteases could be involved in this amoeba's ability to invade the CNS. Our studies are feasible with the availability of the in vitro cultures of the human brain microvascular endothelial cells, which constitute the blood-brain barrier. Here, we report that proteases from an AGE isolate of Acanthamoeba digest ECM and produce blood-brain barrier disruptions and thus may play crucial role(s) in invasion of the brain tissue by amoebae.

\section{Results}

Acanthamoeba keratitis isolate (T4 genotype) exhibited two serine proteases while AGE isolate (TI genotype) exhibited one serine and one metalloprotease

To determine the extracellular proteases of Acanthamoeba, conditioned medium (CM) was used for zymographic assays. Acanthamoeba keratitis isolate (T4 genotype) exhibited two protease bands observed at approximate molecular weights $(\mathrm{Mw})$ of $130 \mathrm{KD}$ and $85 \mathrm{KD}$ (Fig. 1A). However, both protease bands were sensitive to PMSF indicating that Acanthamoeba secrete serine proteases, a finding that is consistent with previous studies (reviewed in [5]). Thus, the remaining studies have been focused on the AGE isolate. In contrast, AGE isolate (T1 genotype) exhibited a $150 \mathrm{KD}$ metalloprotease (inhibited with 1, 10phenanthroline) and a $130 \mathrm{KD}$ serine protease (inhibited by PMSF) (Fig. 1B). Of interest, CM produced from co-cultures of Acanthamoeba and HBMEC exhibited similar protease profiles (data not shown). 
A)

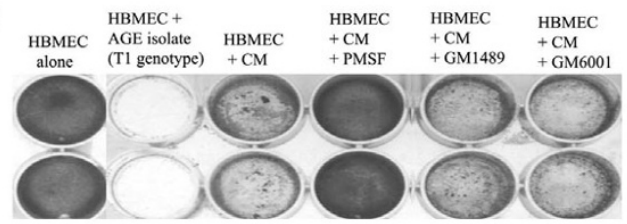

B)

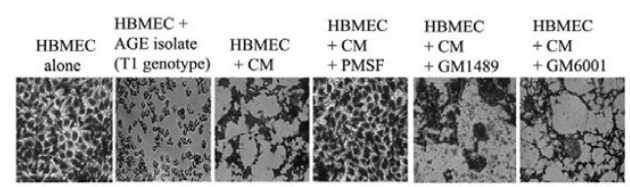

C)

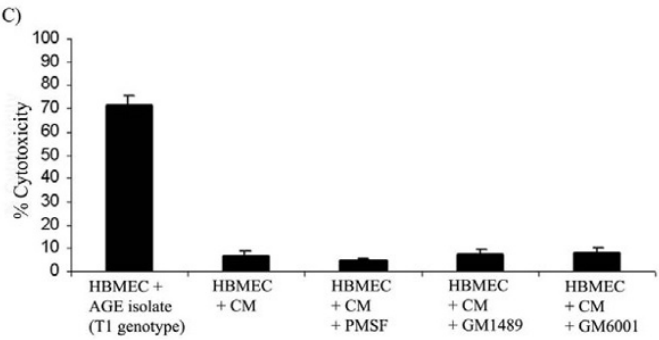

Figure 2

A) Conditioned medium (CM) of the AGE isolate exhibited HBMEC monolayer disruptions. Acanthamoeba (AGE isolate belonging to TI genotype) or their $C M$ were added to human brain microvascular endothelial cells (HBMEC) monolayers as described in "Materials and Methods". A) HBMEC monolayers were stained with hematoxylin to observe monolayer disruptions. HBMEC alone represent HBMEC without parasites and/or CM and were used as controls. Both Acanthamoeba as well as CM exhibited HBMEC monolayer disruptions. Note that PMSF blocked CM-mediated HBMEC monolayer disruptions. In contrast, metalloprotease inhibitors (GM I489 and GM 600I) had no effects on CM-mediated HBMEC monolayer disruptions. B) Micrography of HBMEC cytopathic effects. Micrographs were taken using a phase-contrast microscope, $\times 200$. C) CM of the AGE isolate did not produce HBMEC cytotoxicity. Note that Acanthamoeba (AGE isolate belonging to TI genotype) exhibited more than 70\% HBMEC cytotoxicity, while their CM induced less than 10\% HBMEC cytotoxicity. Results are mean of three experiments performed in duplicates. Error bars represent standard error.

Conditioned medium of the AGE isolate exhibited HBMEC monolayer disruptions but not cytotoxicity

To determine the cytopathic effects of extracellular proteases of AGE isolate (T1 genotype), cytotoxicity assays were performed. Hematoxylin staining of HBMEC monolayers revealed that the AGE isolate as well as its $\mathrm{CM}$ produced total HBMEC monolayer disruptions (Fig. 2A and 2B). The CM-mediated HBMEC monolayer disruptions were completely abolished after pre-incubating the CM with PMSF (Fig. 2A and 2B). Pre-incubations with broad spectrum matrix metalloprotease (MMP) inhibitors, i.e., GM 1489 or GM 6001, had no effect on CM-mediated HBMEC monolayer disruptions (Fig. 2A and 2B). The AGE isolate produced more than 70\% HBMEC cytotoxicity, while its CM exhibited minimal HBMEC cytotoxicity (Fig. 2C). Furthermore, neither broad spectrum MMP inhibitors had an effect on AGE isolate-mediated HBMEC cytotoxicity (data not shown). Similar findings were observed with $\mathrm{AK}$ isolate belonging to T4 genotype (data not shown). Taken together, these data demonstrate that 130 KD serine protease (but not the 150 KD metalloprotease) produced HBMEC monolayer disruptions but not HBMEC cytotoxicity.

\section{The effect of various temperatures and $\mathrm{pH}$ on the extracellular proteases of AGE isolate}

To determine the physiological properties of extracellular proteases of AGE isolate (T1 genotype), zymographic assays were performed using gelatin as substrate and gels incubated at various temperatures and $\mathrm{pH}$. The results revealed that both serine and metalloproteases exhibited optimal activities at neutral pH (Fig. 3). The influence of various temperatures on the proteolytic activities was tested in the temperature intervals from $4{ }^{\circ} \mathrm{C}-65^{\circ} \mathrm{C}$. The optimal activity was observed at temperatures varying from $37^{\circ} \mathrm{C}-50^{\circ} \mathrm{C}$ (Fig. 4), indicating that proteases are also active at physiologically relevant conditions. Of interest, the metalloprotease (approx. Mw. 150 KD) visually appeared to have lost significant activity in zymography during incubation at $65^{\circ} \mathrm{C}$ (Fig. 4).

\section{Both serine (I30 KD) and metalloproteases (I50 KD) of AGE isolate exhibited collagenase and elastinolytic activities}

The fibrillary collagens and elastin are structural proteins that are the major components of the ECM. To determine the ability of serine and metalloproteases to exhibit collagenase activities, zymographic assays were performed using collagen I and III as substrates. Both serine and metalloproteases degraded collagen I and III substrates (Fig. 5) confirming their collagenase activities. Additionally, both proteases degraded elastin (a major constituent of elastic fibers) in the zymographic assays, indicating that proteases from AGE isolate exhibit elastinolytic activities (Fig. 5). Among other substrates, these proteases exhibited degradation of casein and haemoglobin (Fig. 5). 


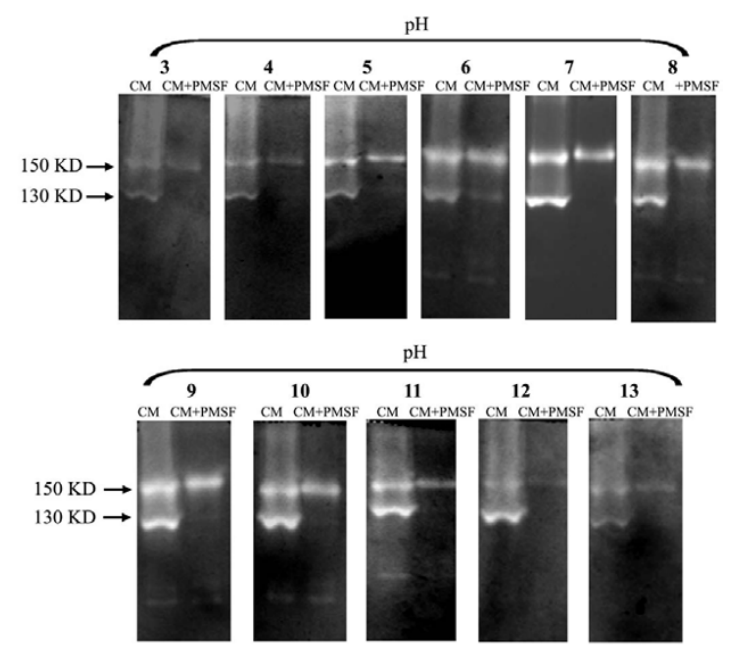

Figure 3

Acanthamoeba (AGE isolate belonging to TI genotype) proteases exhibit optimal activities at neutral $\mathbf{p H}$. To determine the effects of $\mathrm{pH}$, zymographic assays were performed as described in the "Materials and Methods" and gels incubated at various $\mathrm{pHs}$. Note that neutral $\mathrm{pH}$ produced optimal proteolytic activities. Results are representative of three independent experiments. Each lane contained $0.5 \mu \mathrm{g}$ protein.

Both serine (I30 KD) and metalloproteases (I50 KD) of AGE isolate degrade plasminogen

Other modes of ECM degradation are tissue-type and urokinase-type plasminogen activators. The former pathway is mostly involved in fibrinolysis, while the latter is an important modulator in the pathophysiology of neuronal damage [8]. The activated urokinase converts proenzyme, plasminogen into plasmin, a serine protease involved in the ECM destruction by degrading fibrin. In this study, the zymographic assays revealed that both serine and metalloproteases from AGE isolate degraded plasminogen at neutral $\mathrm{pH}$ (Fig. 5).
The metalloprotease activity can be separated from the serine protease activity by ion exchange chromatography In an attempt to obtain purified metalloprotease, FPLC ion-exchange chromatography was employed. The chromatograms in Fig. 6 show at least two major peaks and the corresponding column fractions exhibited protease activity. The eluted protein(s) in the first peak (fractions 10 and 11) contained metalloprotease activity (approx. Mw. 150 KD) (Fig. 6), separated apparently from the serine protease and sensitive to 1,10-phenanthroline on gelatin gel zymography (data not shown). Of interest, the serine protease was observed in later fractions (data not shown).

\section{Discussion}

Acanthamoeba granulomatous encephalitis is a serious CNS infection that almost always results in death. The pathophysiological complications involving the CNS most likely include invasion of the connective tissue, induction of extensive pro-inflammatory responses and neuronal damage leading to brain dysfunction. Thus it is reasonable to predict that Acanthamoeba proteases play important roles in AGE pathogenesis. Our previous studies have shown that Acanthamoeba proteases may play important roles in modulating blood-brain barrier permeability [9]. In this study, we further characterized Acanthamoeba proteases from an AGE isolate and developed methods to separate an extracellular metalloprotease from the extracellular serine protease.

The fact that a $150 \mathrm{KD}$ extracellular metalloprotease is associated with an AGE isolate (and not keratitis isolate) suggests its possible involvement in the CNS pathology. To this end, we studied the properties of AGE isolate proteases on ECM degradation. In healthy brains, ECM comprises a major percent of the normal brain volume [10], which forms the basal lamina around the blood vessels. The ECM is constantly remodelled and provides critical structural and functional support to the neuronal tissue. These properties of ECM are tightly regulated by a family of mostly $\mathrm{Ca}^{2+}$-dependent $\mathrm{Zn}^{2+}$-containing endopeptidases (MMPs) [11,12]. The ECM plays important roles under normal physiological conditions in the development and maintenance of homeostasis in neuronal tissue. However in neurological disease states, ECM may undergo substantial modifications resulting in neuroinflammatory responses. Excessive ECM degradation affects neurovascular structural/functional properties that are highly destructive to the CNS functions. ECM is composed of both collagenous and non-collagenous glycoproteins and proteoglycans $[11,12]$. Here we showed that both the serine and metalloproteases of the AGE isolate exhibits collagen I and collagen III degradation suggesting that these proteases may facilitate amoebic migration into deeper tissues by degrading the ECM. These findings support previous findings which showed that Acanthamoeba 


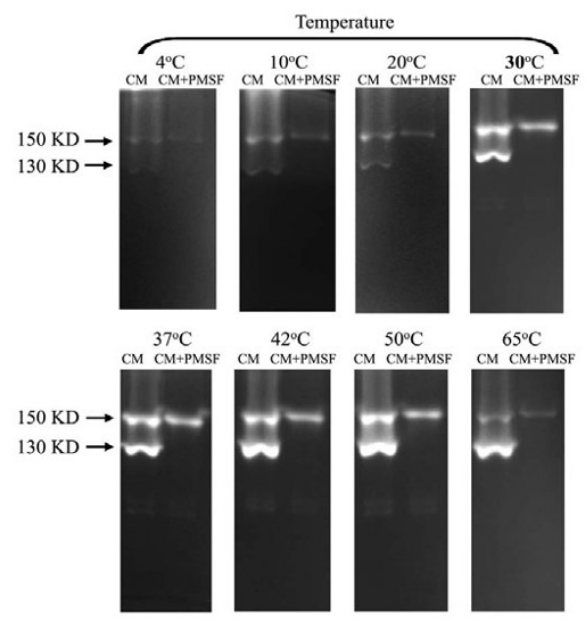

\section{Figure 4}

Acanthamoeba (AGE isolate belonging to TI genotype) proteases exhibit optimal activities at various temperatures. To determine the effects of variable temperatures, zymographic assays were performed as described in the "Materials and Methods" and gels incubated at various temperatures. Note that $37-50^{\circ} \mathrm{C}$ produced optimal proteolytic activities. Results are representative of three independent experiments. Each lane contained $0.5 \mu \mathrm{g}$ protein.

exhibit collagenolytic activities [13]. Furthermore, both proteases exhibited elastinolytic activities. Elastin is the principal structural element that constitutes elastic fibres and is an important component of connective tissue. Previous findings have shown that elastase destroys ECM, which increases blood-brain barrier permeability resulting in the brain injury [14-16]. For example, injection of elastase into the cerebrospinal fluid (CSF) opened the blood-brain barrier in newborn piglets [17]. Our findings suggest that proteases of AGE isolate may play a similar role.

In addition to the aforementioned, the urokinase plasminogen activator system plays an important role in various neuronal diseases involving the CNS inflammation and/or pathology. For example, in bacterial meningitis, the uPA (urokinase-type plasminogen activator) or tPA (tissue-type plasminogen activator) are known to convert plasminogen, that is abundant in brain [18] into plasmin, which destroys ECM directly by degrading fibrin or by activating MMPs. We observed that both serine and metalloproteases directly degraded pro-enzyme, plasminogen suggesting that pathogenesis of AGE may involve the uPA/ tPA system. Injections of plasmin results in increased capillary permeability [19]. At present, the precise role of the 150 KD metalloproteases remains unclear.

Our findings that CM-mediated HBMEC monolayer disruptions can be inhibited in the presence of PMSF suggest that the metalloprotease may not be responsible for HBMEC monolayer disruptions and may play a role in post-CNS invasion by amoebae. To this end, we have successfully developed methods for the separation of the metalloprotease from the serine protease of the AGE isolate, which should prove useful for subsequent purification and biochemical characterizations of this novel protease as well as to determine its role in vivo.

It is important to note that we only observed two proteases in our zymographic assays. However, the experimental conditions involved SDS detergent and there may be other proteases that are SDS-sensitive. Also, the identification of $130 \mathrm{KD}$ serine protease is not novel. Previous studies have characterized a similar protease from an AK isolates [20-22] and again in the present study we observed $130 \mathrm{KD}$ serine protease from an $\mathrm{AK}$ isolate belonging to T4 genotype. Thus it is reasonable to presume that the $130 \mathrm{KD}$ serine protease is similar in both $\mathrm{T} 1$ and T4 isolates. Taken together, these studies have characterized the properties of a novel metalloprotease that only is produced by the AGE isolate. Future studies will precise determine its role in the virulence of AGE. Of interest, metalloproteases have been identified as important virulence factors in various opportunistic pathogens including Vibrio vulnificus [23], Pseudomonas aeruginosa [24], and Aspergillus fumigatus [25].

\section{Conclusion}

In summary, these data show that both serine and metalloproteases secreted by AGE isolate (T1 genotype), exhibit properties to degrade ECM, which may facilitate amoebic invasion of the deeper lying tissues of the CNS. If future studies confirm that these proteases are indeed important in the pathology of AGE then they could be potential targets for the rationale development of therapeutic interventions.

\section{Methods}

All chemicals were purchased from Sigma (Poole, Dorset, UK), unless otherwise stated. 


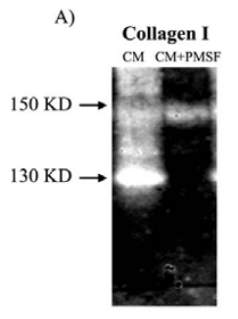

B)
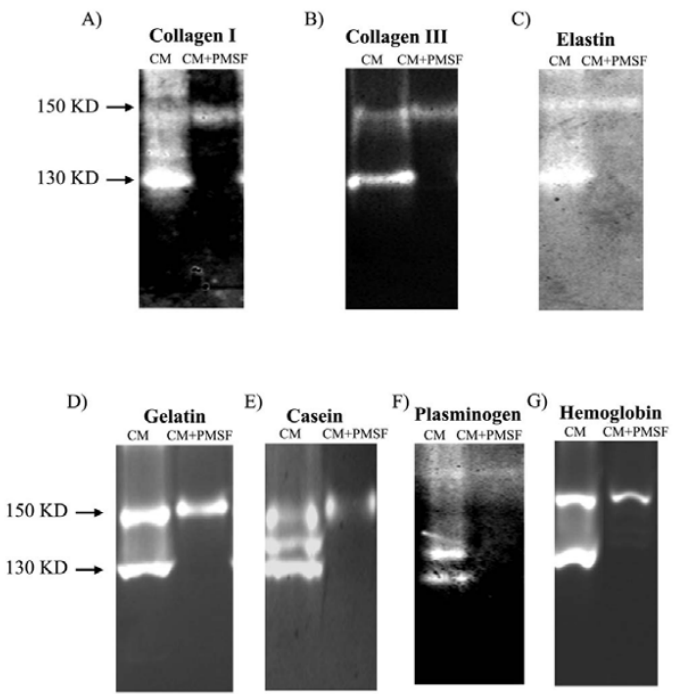

\section{Figure 5}

Acanthamoeba (TI) proteases degrade various protein substrates including extracellular matrix (ECM). Zymographic assays were performed using various substrates that constitute ECM or involved in ECM degradation. Note that proteases of AGE isolate degraded collagen I (A), collagen III (B), elastin (C) and plasminogen (F). In addition, both proteases degraded gelatin (D), casein (E) and haemoglobin (G). CM is conditioned medium plus or minus the serine protease inhibitor, PMSF. Results are representative of three independent experiments.

\section{Acanthamoeba cultures}

Two isolates of Acanthamoeba were used in the present study: 1) A. castellanii (ATCC 50494) belonging to T1 genotype was isolated from an AGE patient, and 2) A. castellanii (ATCC 50492)belonging to T4 genotype was isolated from a keratitis patient. For simplicity, the former is referred to as the AGE isolate and the latter is referred to as the AK isolate. Both isolates were grown in tissue culture flasks in PYG medium [proteose peptone $0.75 \%(\mathrm{w} /$ $\mathrm{v})$, yeast extract $0.75 \%(\mathrm{w} / \mathrm{v})$ and glucose $1.5 \%(\mathrm{w} / \mathrm{v})]$ at $30^{\circ} \mathrm{C}$ and the medium was refreshed $17-20 \mathrm{~h}$ prior to experiments as previously described [26]. This resulted in $>95 \%$ Acanthamoeba in the trophozoite forms.

\section{Human brain microvascular endothelial cell cultures}

The primary brain microvascular endothelial cells were isolated from human tissue and purified by fluorescent activated cell sorting (FACS). The purified cells were tested for endothelial characteristics, such as expression of endothelial markers, F-VIII, carbonic anhydrase IV and uptake of acetylated low density lipoprotein (AcLDL), indicating their endothelial origin and expression of gamma-glutamyl transpeptidase, indicating the brain origin (27). HBMEC were grown in RPMI-1640 containing $10 \%$ foetal bovine serum, $10 \%$ NuSerum, $2 \mathrm{mM}$ glutamine, $1 \mathrm{mM}$ pyruvate, penicillin $(100 \mathrm{U} / \mathrm{ml})$, streptomycin $(100 \mathrm{U} / \mathrm{ml})$, non-essential amino acids and vitamins at $37^{\circ} \mathrm{C}, 5 \% \mathrm{CO}_{2}$ as previously described $[27,28]$.

\section{Cytotoxicity assays}

To examine the cytopathic potential of Acanthamoeba isolates, cytotoxicity assays were performed as previously described [26]. Briefly, HBMEC were grown to confluent monolayers in 24-well plates. Acanthamoeba $\left(5 \times 10^{5}\right.$ amoebae/well) were incubated with HBMEC monolayers in serum free medium (RPMI 1640 containing $2 \mathrm{mM}$ glutamine, $1 \mathrm{mM}$ pyruate and non-essential amino acids) in plates incubated at $37^{\circ} \mathrm{C}$ in $5 \% \mathrm{CO}_{2}$. The HBMEC monolayers were observed periodically under a phasecontrast microscope for cytopathic effects for up to $24 \mathrm{~h}$. After $24 \mathrm{~h}$, the supernatants (termed conditioned medium, CM) were collected, and the monolayers were fixed and stained with hematoxylin to visualize HBMEC monolayer disruptions. In addition, supernatants were examined for HBMEC cytotoxicity by measuring lactate dehydrogenase (LDH) release (cytotoxicity detection kit; Roche Applied Science, Lewes, East Sussex, UK). Briefly, $\mathrm{CM}$ of co-cultures of Acanthamoeba and HBMEC were assessed for the presence of $\mathrm{LDH}$, the release of which is considered as an estimate of cell death. The percentage LDH release was calculated as follows: [LDH activity in experimental sample (measured by optical density at 590 $\mathrm{nm}$ ) - LDH activity in control samples/total LDH activity release $-\mathrm{LDH}$ activity in control samples $\times 100=\%$ cytotoxicity). Control samples were obtained from HBMEC or Acanthamoeba incubated alone. Total LDH activity release was determined by total HBMEC lysis with $1 \%$ Triton X100 for $30 \mathrm{~min}$ at $37^{\circ} \mathrm{C}$.

To determine the cytopathic effects of extracellular proteases released from intact Acanthamoeba, CM was produced by inoculating Acanthamoeba in serum free medium described above for $24 \mathrm{~h}$. Next day, Acanthamoeba free supernatants, i.e., $\mathrm{CM}$ was collected by centrifugation and used for cytotoxicity assays as described above.

Some cytotoxicity assays were performed in the presence or absence of broad spectrum matrix metalloprotease (MMP) inhibitors, i.e., GM 6001 and GM 1489 (Merck 
A)
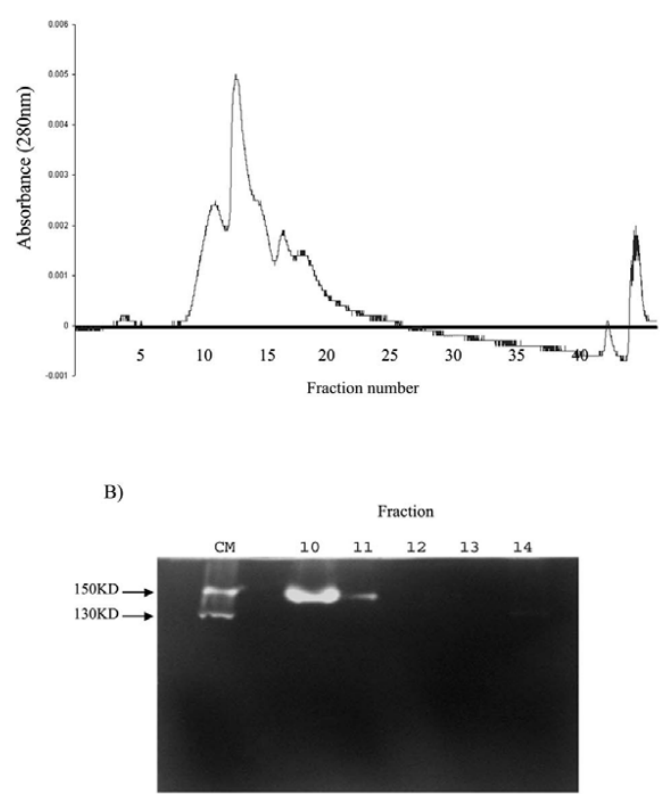

\section{Figure 6}

Separation of metalloprotease by ion-exchange chromatography. Proteins in CM from the AGE isolate were precipitated and fractionated using FPLC ion-exchange chromatography. A) A representative chromatogram showing a typical elution profile is shown. The collected fractions were tested for protease activity, and B) shows that fractions 10 and II (in the first major UV-absorbing peak) exhibited only metalloprotease activity (approx. Mw. I50 KD). Results are representative of three experiments.

Biosciences Ltd. Nottingham, England) or phenylmethanesulfonyl fluoride (PMSF), an irreversible inhibitor of serine proteases. Briefly, the protease inhibitors (PMSF, 2 mM final conc.; GM 6001 and GM 1489, $5 \mu \mathrm{M}$ final conc.) were added to $\mathrm{CM}$ for $30 \mathrm{~min}$ and then mixtures were added to HBMEC monolayers.

\section{Zymography}

Zymographic assays were performed to visualize and begin to characterize Acanthamoeba extracellular proteases as previously described [29]. Briefly, CM was mixed (1:1) with sample buffer (containing 4\% sodium dodecyl sulfate (SDS) but without $\beta$-mercaptoethanol) and electrophoresed on SDS-polyacrylamide gels (SDS-PAGE) containing gelatin $(1 \mathrm{mg} / \mathrm{mL})$. After electrophoresis, gels were soaked in $2.5 \%$ Triton X-100 (w/v) for $60 \mathrm{~min}$ to remove the SDS. Finally, the gels were incubated in a developing buffer ( $50 \mathrm{mM}$ Tris- $\mathrm{HCl}, \mathrm{pH} 7.5$, containing $10 \mathrm{mM} \mathrm{CaCl}_{2}$ ) at $37^{\circ} \mathrm{C}$ overnight, rinsed, and stained with Coomassie brilliant blue. Areas of gelatin digestion indicating protease activities are seen as non-staining regions in the gel. In some experiments, samples were pretreated with PMSF ( $2 \mathrm{mM}$ final concentration), or 1, 10phenanthroline (10 $\mathrm{mM}$ final concentration) for $30 \mathrm{~min}$. Because 1, 10-phenanthroline is a reversible inhibitor, it was also included in the developing buffer.

To determine the optimum temperature for Acanthamoeba protease activities, gels were incubated at $4^{\circ} \mathrm{C}, 10^{\circ} \mathrm{C}$, $20^{\circ} \mathrm{C}, 30^{\circ} \mathrm{C}, 37^{\circ} \mathrm{C}, 42^{\circ} \mathrm{C}, 50^{\circ} \mathrm{C}$ and $65^{\circ} \mathrm{C}$. For the determination of optimum $\mathrm{pH}$, gels were incubated at $\mathrm{pH}$ ranging from 3 - 13. For pH 3 and 4, developing buffers were prepared using citrate-phosphate buffer $(70 \mathrm{mM}$ sodium citrate and $60 \mathrm{mM}$ sodium phosphate) containing $10 \mathrm{mM}$ $\mathrm{CaCl}_{2}$; for $\mathrm{pH} 5$ and 6 , developing buffers were prepared using N- [2-morpholino] ethanesulfonic acid (MES) containing $10 \mathrm{mM} \mathrm{CaCl}_{2}$; and for $\mathrm{pH} 7$ - 13, developing buffers were prepared using $50 \mathrm{mM}$ Tris containing $10 \mathrm{mM}$ $\mathrm{CaCl}_{2}$.

In some experiments, we used extracellular matrix (ECM) proteins including collagen I (obtained from bovine achilles tendon; $1 \mathrm{mg} / \mathrm{mL}$ final conc.) dissolved in buffer A $(50 \mathrm{nM}$ TES \{N-tris [hydroxymethyl]methyl-2-aminoethane-sulfonic acid\}, pH 7.4 plus $0.36 \mathrm{mM} \mathrm{CaCl}_{2}$ ), collagen III (calf skin; $1 \mathrm{mg} / \mathrm{mL}$ final conc.) dissolved in buffer $\mathrm{B}$ (distilled $\mathrm{H}_{2} \mathrm{O}, \mathrm{pH} 3$ using acetic acid), elastin (bovine neck ligament; $1 \mathrm{mg} / \mathrm{mL}$ final conc.) dissolved in buffer C (200 mM Tris, $\mathrm{pH} 8.8$ ) and plasminogen (rabbit plasma; $1 \mathrm{mg} / \mathrm{mL}$ final conc.) dissolved in buffer D (20 $\mathrm{mM}$ lysine, pH 7) as substrate for Acanthamoeba proteases. In addition we used casein (bovine milk; $1 \mathrm{mg} / \mathrm{mL}$ final conc.) dissolved in buffer E $(60 \mathrm{mM}$ Tris, $90 \mathrm{mM} \mathrm{NaCl}$, pH7.5) and haemoglobin (bovine erthyrocytes; $1 \mathrm{mg} / \mathrm{mL}$ final conc.) dissolved in distilled $\mathrm{H}_{2} \mathrm{O}$ with gentle heat as substrate for Acanthamoeba proteases. All substrates were dissolved according to manufacturer's instructions (i.e., Sigma Labs).

\section{Ammonium sulphate precipitation}

In an attempt to purify extracellular proteases of Acanthamoeba, (ca. $500 \mathrm{~mL}$ ), CM was produced and proteins precipitated by adding ammonium sulphate $(0.7 \mathrm{~g} / \mathrm{mL})$ slowly to the $\mathrm{CM}$ while stirring at $4^{\circ} \mathrm{C}$ to achieve $70 \%$ saturation. The mixture was stirred for an additional $25 \mathrm{~min}$. The precipitate was recovered by centrifugation $(10,000 \times$ $g$ for $10 \mathrm{~min}$ at $4^{\circ} \mathrm{C}$ ) and dissolved in $500 \mu \mathrm{l}$ of $0.02 \mathrm{M}$ ammonium acetate buffer, $\mathrm{pH}$ 6.5. The concentrated pro- 
teins were dialysed against HPLC grade $\mathrm{H}_{2} \mathrm{O}$ overnight to remove residual ammonium sulphate.

\section{lon-exchange chromatography}

The dialysed precipitated proteins were applied to a DEAE-Sepharose fast flow beads column (16 cm length, 2 $\mathrm{cm}$ diameter) (Amersham Biosciences). The column was equiliberated with $100 \mathrm{~mL}$ of $0.02 \mathrm{M}$ ammonium acetate buffer, pH 6.5 at a flow rate of $4 \mathrm{~mL}$ per min. Using a fast performance liquid chromatography system (Biorad, Hemel Hempstead, UK), proteins were eluted in $0.02 \mathrm{M}$ ammonium acetate with a 0 to $1.0 \mathrm{M} \mathrm{NaCl}$ gradient. Proteins in the eluted fractions $(6 \mathrm{~mL})$ were freeze dried for long term storage. The lyophilised fractions were reconstituted in $1 \mathrm{~mL}$ of $50 \mathrm{mM}$ Tris- $\mathrm{HCl}, \mathrm{pH} 6.8$ and tested for protease activities using zymographic assays.

\section{Acknowledgements}

This work was partially supported by grants from Faculty Research Fund, Central Research Fund, University of London, British Council for Prevention of Blindness and the Royal Society.

\section{References}

I. Martinez AJ, Visvesvara GS: Free-living, amphizoic and opportunistic amebas. Brain Pathol 1997, 7:583-598.

2. Martinez AJ: Free-living Amebas: Natural History, Prevention, Diagnosis, Pathology and treatment of disease, CRC Press, Boca Raton, Florida. 1985: 156.

3. Marciano-Cabral F, Cabral G: Acanthamoeba spp. as agents of disease in humans. Clin Microbiol Rev 2003, 16:273-307.

4. Schuster FL, Visvesvara GS: Free-living amoebae as opportunistic and non-opportunistic pathogens of humans and animals. Int J Parasitol 2004, 34: 100I-I027.

5. Khan NA: Pathogenesis of Acanthamoeba infections. Microb Pathogen 2003, 34:277-285.

6. Alsam S, Kim KS, Stins M, Rivas AO, Sissons J, Khan NA: Acanthamoeba interactions with human brain microvascular endothelial cells. Microb Pathogen 2003, 35:235-24I.

7. Klemba M, Goldberg DE: Biological roles of proteases in parasitic protozoa. Annu Rev Biochem 2002, 7 I:275-305.

8. Busch E, Kruger K, Fritze K, Allegrini PR, Hoehn-Berlage M, Hossmann KA: Blood-brain barrier disturbances after rt-PA treatment of thromboembolic stroke in the rat. Acta Neurochir 1997, 70:206-208.

9. Alsam S, Sissons J, Jayasekera S, Khan NA: Extracellular proteases of Acanthamoeba castellanii (encephalitis isolate belonging to TI genotype) contribute to increased permeability in an in vitro model of the human blood-brain barrier. J Infect 2005, 5I:150-I56.

10. Gladson CL: The extracellular matrix of gliomas: modulation of cell function. J Neuropathol Exp Neurol 1999, 58: 1029-1040.

11. Lukes A, Mun-Bryce S, Lukes M, Rosenberg GA: Extracellular matrix degradation by metalloproteinases and central nervous system diseases. Mol Neurobiol 1999, 19:267-284.

12. Rosenberg GA: Matrix metalloproteinases in neuroinflammation. Glia 2002, 39:279-29I.

13. He YG, Neiderkorn JY, McCulley JP, Stewart GL, Meyer DR, Silvany $\mathrm{R}$, Doughtery J: In vivo and in vitro collagenolytic activity of Acanthamoeba castellanii. Invest Opthal Visual Sci 1990, 31:2235-2240.

14. Janoff A: Elastase in tissue injury. Annu Rev Med 1985, 36:207-2I6.

15. Rudolphus A, Stolk J, van Twisk C, van Noorden CJ, Dijkman JH, Kramps JA: Detection of extracellular neutrophil elastase in hamster lungs after intratracheal instillation of $E$. coli lipopolysaccharide using a fluorogenic, elastase-specific, synthetic substrate. Am J Pathol 1992, I 41:153-160.

16. Yasui S, Nagai A, Aoshiba K, Ozawa Y, Kakuta Y, Konno K: A specific neutrophil elastase inhibitor (ONO-5046.Na) attenu- ates LPS-induced acute lung inflammation in the hamster. Eur Respir J 1995, 8: I293-1299.

17. Temesvari P, Abraham CS, Gellen J Jr, Speer CP, Kovacs J, Megyeri P: Elastase given intracisternally opens blood-brain barrier in newborn piglets. Biol Neonate 1995, 67:59-63.

18. Soreq $\mathrm{H}$, Miskin R: Plasminogen activator in the rodent brain. Brain Res 198I, 216:361-374.

19. Armao D, Kornfeld M, Estrada EY, Grossetete M, Rosenberg GA: Neutral proteases and disruption of the blood-brain barrier in rat. Brain Res 1997, 5:259-264.

20. Leher H, Silvany R, Alizadeh H, Huang J, Niederkorn JY: Mannose induces the release of cytopathic factors from Acanthamoeba castellanii. Infect Immun 1998, 66:5-10.

21. Hurt M, Niederkorn J, Alizadeh H: Effects of mannose on Acanthamoeba castellanii proliferation and cytolytic ability to corneal epithelial cells. Invest Ophthalmol Vis Sci 2003, 44:3424-343I.

22. Hurt M, Neelam S, Niederkorn J, Alizadeh H: Pathogenic Acanthamoeba spp secrete a mannose-induced cytolytic protein that correlates with the ability to cause disease. Infect Immun 2003, 71:6243-6255.

23. Miyoshi N, Shimizu C, Miyoshi S, Shinoda S: Purification and characterization of Vibrio vulnificus protease. Microbiol Immunol 1987, 3 I: 13-25.

24. Wretlind $B$, Pavlovskis OR: Pseudomonas aeruginosa elastase and its role in pseudomonas infections. Rev Infect Dis 1983, 5:S998-1004.

25. Markaryan A, Morozova I, Yu H, Kolattukudy PE: Purification and characterization of an elastinolytic metalloprotease from Aspergillus fumigatus and immunoelectron microscopic evidence of secretion of this enzyme by the fungus invading the murine lung. Infect Immun 1994, 62:2 I 49-2I 57 .

26. Sissons J, Kim KS, Stins M, Jayasekera S, Alsam S, Khan NA: Acanthamoeba castellanii induces host cell death via a phosphatidylinositol 3-kinase-dependent mechanism. Infect Immun 2005, 73:2704-2708.

27. Stins MF, Gilles F, Kim KS: Selective expression of adhesion molecules on human brain microvascular endothelial cells. J Neuroimmunol 1997, 76:8I-90.

28. Jayasekera S, Matin A, Sissons J, Maghsood AH, Khan NA: Balamuthia mandrillaris stimulates interleukin- 6 release in primary human brain microvascular endothelial cells via a phosphatidylinositol 3-kinase-dependent pathway. Microb Infect 2005, 7:|1345-|35|.

29. Khan NA, Jarroll EL, Panjwani N, Cao Z, Paget TA: Proteases as markers for differentiation of pathogenic and nonpathogenic species of Acanthamoeba. J Clin Microbiol 2000, 38:2858-286I.
Publish with BioMed Central and every scientist can read your work free of charge

"BioMed Central will be the most significant development for disseminating the results of biomedical research in our lifetime. "

Sir Paul Nurse, Cancer Research UK

Your research papers will be:

- available free of charge to the entire biomedical community

- peer reviewed and published immediately upon acceptance

- cited in PubMed and archived on PubMed Central

- yours - you keep the copyright

Submit your manuscript here:

http://www.biomedcentral.com/info/publishing_adv.asp
BioMedcentral 